\title{
Entgeltgleichheit und Begrenzung der Flexibilisierung geringfügiger Beschäftigung durch kollektivrecht- liche Regelungen
}

Geringfügig Beschäftigte genießen bei Steuern und Abgaben zwar Privilegien, arbeitsrechtlich erleiden sie jedoch häufig eklatante Nachteile. Rechte der Beschäftigten werden flächendeckend missachtet, so beispielsweise bei der Entgeltfortzahlung im Krankheitsfall, dem bezahlten Urlaub oder der Höhe der Entlohnung. Der Beitrag analysiert die gesetzlichen Grundlagen des flexiblen Einsatzes bis hin zur Arbeit auf Abruf und die Regelungen zur Vermeidung der Diskriminierung geringfügig Beschäftigter. Die Betriebs- und Tarifvertragsparteien verfügen zudem über rechtliche Spielräume, die sie zum Schutz von Minijobbern einsetzen können.

REINGARD ZIMMER

\section{Einleitung}

Im Dezember 2010 gab es in Deutschland insgesamt 7,38 Mio. geringfügig Beschäftigte (sogenannte Minijobber) mit einer Entlohnung von bis zu $400 €$ monatlich, knapp zwei Drittel sind Frauen (BA 2011). Geringfügige Beschäftigung ist seit Jahren in der Kritik, da sie aufgrund des geringen Ertrags nicht Existenz sichernd ist, sondern die Abhängigkeit von einem Partner bzw. einer Partnerin oder von staatlichen Transferleistungen voraussetzt. So mussten Mitte 2010 rund 660.000 geringfügig Entlohnte ihr Entgelt durch Arbeitslosengeld II „aufstocken“, womit sie die größte Gruppe der „Aufstocker“ darstellen (BA 2010). Zudem erwerben Minijobber weder eigenen Versicherungsschutz in der Kranken- und Pflegeversicherung, noch wird ein Anspruch auf Arbeitslosengeld I vermittelt. Rentenrechtlich erwächst aufgrund der anteiligen Beitragszahlung nur eine sehr geringe Anwartschaft, sodass Altersarmut vorprogrammiert ist. ${ }^{0}$ Diese prekäre Beschäftigungsform belastet daher aufgrund der notwendigen staatlichen Transferleistungen die sozialen Sicherungssysteme.

Geringfügig Beschäftigten wird nicht selten ein hohes Maß an Flexibilität abverlangt, denn sie können oftmals nur sehr begrenzt Dauer, Lage und Verteilung ihrer Arbeitszeit beeinflussen (Zimmer 2010, S. 302). Eine Untersuchung der Praktiken des sächsischen Einzelhandels zeigt, dass Minijobber häufig lediglich für kürzere Phasen eingesetzt wer- den, oft nur für zwei Stunden, zudem werden sie oftmals erst einen Tag vorher oder gar am selben Tag über die Einsatzplanung informiert. Ähnlich kurzfristig werden geringfügig Beschäftigte wieder nach Hause geschickt, wenn ihr Arbeitseinsatz nicht mehr benötigt wird (Benkhoff/Hermet 2008, S. 22). Untersuchungen zeigen, dass eine solche Praxis kein Einzelfall ist, arbeitsrechtliche Vorschriften werden vielmehr in signifikantem Ausmaß verletzt. Insbesondere leisten Arbeitgeber häufig keine Entgeltfortzahlung im Krankheitsfall. Bei Krankheit, Urlaub und gesetzlichen Feiertagen werden die Einsätze geringfügig Beschäftigter durch flexible Einsatzplanung kurzfristig außerhalb solcher Zeiten gelegt, sodass die eigentlich erforderliche Entgeltfortzahlung umgangen wird. Von Sonderzahlungen sind Minijobber in der Regel ausgenommen (Benkhoff/Hermet 2008, S. 20 f.). Die Beschäftigten selbst sind häufig nicht über ihre Rechte informiert oder wagen es nicht, sie durchzusetzen. ${ }^{\circledR}$ Das

(1) Laut Berechnungen von Waltermann (2010, S. 21) führt ein Entgelt von $400 €$ über 45 Beitragsjahre (!) zu einer Rente von $143,45 €$ West/127,26 € Ost.

(2) So haben nach Angaben der Minijobzentrale im Jahr 2004 nur 7,6\% der in Kleinbetrieben geringfügig Beschäftigten Entgeltfortzahlung im Krankheitsfall beantragt, während der Prozentsatz bei sozialversicherungspflichtig Beschäftigten $56 \%$ betrug (Benkhoff/Hermet 2008, S. 7). 
Konstrukt abgabenprivilegierter geringfügiger Beschäftigung ist arbeitsrechtlich offensichtlich insbesondere hinsichtlich der Arbeitszeitverteilung und der Entlohnung missbrauchsanfällig.

Geringfügige Beschäftigung wird zudem überwiegend zu Niedriglöhnen ausgeübt: Mit durchschnittlich 8,98€ brutto ist der Stundenlohn von Minijobbern nur halb so hoch wie der von Arbeitnehmern, die in einem „Normalarbeitsverhältnis“ ${ }^{(3}$ stehen $(18,04 €$, Statistisches Bundesamt 2009, S. 4). ${ }^{\circledR}$ Bei Dienstleistungsunternehmen liegt der Stundenlohn durchschnittlich gar nur bei $5 €$ (Nebe/Bernhardt 2011, S. 3). Hinzu kommt, dass ostdeutsche geringfügig beschäftigte Frauen offenbar Überstunden meist ohne Bezahlung ableisten (Wolf 2002, S. 643 ff.). Die vom Arbeitgeber zu tragenden Pauschalabgaben in Höhe von knapp $30 \%$ scheinen unzulässigerweise auf die Minijobber abgewälzt zu werden, zum Teil werden (nur) mit dieser Beschäftigungsgruppe auch Nettolohnabreden getroffen.

Dieser Beitrag beschäftigt sich mit dem Schutz von geringfügig Beschäftigten vor Diskriminierung. Insbesondere wird der Frage der Entgeltdiskriminierung nachgegangen, und ob Nettolohnvereinbarungen ausschließlich für geringfügig Beschäftigte zulässig sind (Abschnitt 2.1). Darüber hinaus werden die rechtlichen Rahmenbedingungen der Arbeitszeitflexibilisierung bei Minijobbern analysiert (2.2) und Handlungsmöglichkeiten der Betriebs- (3.1) und Tarifvertragsparteien (3.2) aufgezeigt.

\section{Rechtliche Fragestellungen}

\subsection{Diskriminierungsschutz für geringfügig Beschäftigte}

Auch wenn sozialrechtlich privilegiert, handelt es sich bei geringfügiger Beschäftigung um „normale“ Teilzeitarbeitsverhältnisse ( $\$ 2$ Abs. 2 Teilzeit- und Befristungsgesetz), für die $₫ 4$ Abs. 1 S. 1 TzBfG jegliche Diskriminierung verbietet, sofern nicht eine sachliche Rechtfertigung für die Ungleichbehandlung gegeben ist. Dieses Diskriminierungsverbot ist nicht dispositiv, sondern zwingendes Recht (22 TzBfG), sodass entgegenstehende Regelungen oder Maßnahmen gem. $\$ 134$ Bürgerliches Gesetzbuch (BGB) unwirksam sind. Geringfügig Beschäftigte haben folglich - wie alle anderen Arbeitnehmerinnen und Arbeitnehmer - Anspruch auf Entgeltfortzahlung im Krankheitsfall ( $\$ 3$ Abs. Entgeltfortzahlungsgesetz) oder für Arbeitszeit, die wegen eines gesetzlichen Feiertages ausfällt. Auch im Fall von Schwangerschaft ist während der Zeit von Beschäftigungsverboten nach dem Mutterschutzgesetz (MuSchG) sowie der Zeit der Mutterschutzfristen das Entgelt fortzuzahlen, zudem greift das in $\$ 9$ Abs. 1 MuSchG normierte Kündigungsverbot. Ebenso besteht ein (anteiliger) Anspruch auf mindestens 24 Werktage Urlaub ( $\$ 3$ Abs. 1 Bundesurlaubsgesetz) sowie auf (anteiliges) Urlaubs- und Weihnachtsgeld (EuGH vom 09.09.1999, C-281/97; 22.04.2010, C-486/08).

\subsubsection{Entgeltdiskriminierung im Minijob}

Obwohl der Pro-rata-temporis-Grundsatz aus $₫ 4$ Abs. 1 S. 2 TzBfG normiert, dass Minijobber als Teilzeitbeschäftigte mindestens in dem Umfang zu entlohnen sind, der dem Anteil ihrer Arbeitszeit im Vergleich zu Vollzeitbeschäftigten entspricht, erhalten sie in der Praxis eine deutlich unterdurchschnittliche Entlohnung (vgl. den Beitrag von Voss/Weinkopf in diesem Heft). Wird ihnen ein geringerer Stundenlohn bezahlt als vergleichbaren Vollzeitbeschäftigten (oder hypothetischen Vergleichspersonen), so ist eine direkte Diskriminierung wegen der Teilzeitarbeit im Sinne von $₫ 4$ Abs. 1 TzBfG gegeben. Umstritten ist allerdings, ob sachliche Gründe eine Ungleichbehandlung in Bezug auf das Entgelt rechtfertigen können. Ausgehend vom Wortlaut verneint ein Teil der Literatur die Möglichkeit der sachlichen Rechtfertigung in Bezug auf Ungleichbehandlungen beim Entgelt und anderen geldwerten Leistungen. ${ }^{\ominus}$

Rechtsprechung und ein Teil des Schrifttums gehen jedoch von der Möglichkeit einer sachlichen Rechtfertigung von Ungleichbehandlung beim Entgelt aus. Sie ordnen Satz 1 und 2 in $\$ 4$ Abs. 1 TzBfG als einheitliche Regelung ein und erläutern, Satz 2 solle lediglich verdeutlichen, dass die Entlohnung mindestens proportional zu gewähren ist. $^{\ominus}$ Auch wenn der Wortlaut eindeutig scheint, verdeutlicht doch die Gesetzesbegründung, dass der Pro-ratatemporis-Grundsatz nur dann zur Anwendung kommen soll, wenn dies angemessen ist (BT-Drs. 14/4374, S. 15). Der Gesetzgeber ging somit erkennbar von der Möglichkeit der sachlichen Rechtfertigung einer Ungleichbehandlung (auch) bei der Entlohnung aus. Der sachliche Grund für eine unterschiedliche Entlohnung darf jedoch nicht an der geringeren Arbeitszeit anknüpfen, sondern muss auf andere Gründe wie beispielsweise Qualifikation ge-

3 Das „Normalarbeitsverhältnis" wird definiert als unbefristete, sozialversicherungspflichtige und abhängige Vollzeitbeschäftigung.

$(4 \mathrm{Vgl}$. zum Niedriglohnrisiko auch Pfeifer 2007, S. 67 ff.; Kalina/Weinkopf 2008, S. 8 sowie Brehmer/Seifert 2008, S. $501 \mathrm{ff}$.

5 Vgl. exemplarisch etwa Buschmann et al. 2001, § 4TzBfG Rn. 43; Däubler 2001, S. 218; Richardi et al. 2009, § 161, Rn. 105 ff.; Nielebock 2001, S. 76; Rolfs 2001, S. 131; Schiek 2002, S. 36.

6 Vgl. Bundesarbeitsgericht (BAG) vom 05.11.2003 (5 AZR 8/03), 24.09.2008 (6 AZR 657/07), 05.08.2009 (10 AZR 634/08) sowie aus dem Schrifttum Thüsing 2005, S. 121 Hromadka 2001, S. 675; Kliemt 2001, S. 69; Preis 2011a, Rn. 11f.; Laux/Schlachter 2011, § 4TzBfG, Rn 54; Boecken/ Joussen 2010, § 4TzBfG, Rn. 47 sowie Meinel et al. 2009, § $4 \mathrm{TzBfG}, \mathrm{Rn} .42$. 
gründet werden. Die soziale Lage von Teilzeitbeschäftigten hingegen vermag keinen Rechtfertigungsgrund für eine niedrigere Entlohnung darzustellen, da kein „Soziallohn“, sondern ein Äquivalent für die geleistete Arbeit geschuldet wird. ${ }^{\circ}$

\subsubsection{Nettolohnvereinbarungen ausschließlich für geringfügig Beschäftigte}

Vergleiche hinsichtlich der Lohnhöhe abhängig Beschäftigter setzen üblicherweise am Bruttolohn an, da der Nettolohn abhängig von subjektiven Faktoren wie der Steuerklasse ist. Aus diesem Grund wird sowohl in Individualarbeitsverträgen als auch in Tarifverträgen üblicherweise auf den Bruttolohn abgestellt, wenngleich Nettolohnabreden in Ausnahmefällen existieren. Fraglich ist jedoch, ob die Zahlung von Nettolohnsätzen ausschließlich an die Gruppe der Minijobber zulässig ist. Eine solche Regelung findet sich nicht nur in individuellen Lohnvereinbarungen, sondern bis zum Frühjahr 2011 auch im Tarifvertrag zwischen dem Bundesverband deutscher Dienstleistungsunternehmen und der sogenannten Gewerkschaft DHV. ${ }^{\mathbf{8}}$ Dies könnte nicht nur eine Diskriminierung geringfügig Beschäftigter, sondern auch eine (unzulässige) Umgehung sozialrechtlicher Bestimmungen darstellen. Gemäß $\$ 249$ b Sozialgesetzbuch (SGB) $\mathrm{V}$ sind die Sozialabgaben bei geringfügiger Beschäftigung ausschließlich vom Arbeitgeber zu entrichten, Gleiches schreibt $\$ 168$ Abs. 1 Nr. 1b SGB VI für die Rentenversicherung vor. Da $\$ 32$ SGB I zudem normiert, dass privatrechtliche Vereinbarungen nichtig sind, wenn sie zum Nachteil der Leistungsberechtigten von den Vorschriften des SGB abweichen, ist das Abwälzen der Sozialabgaben auf geringfügig Beschäftigte nicht zulässig. ${ }^{\ominus}$ Lediglich die Pauschalsteuer in Höhe von $2 \%$ darf nach $₫ 40$ a Abs. 2 Einkommensteuergesetz (EStG) auf Arbeitnehmer verlagert werden. ${ }^{\circledR}$

Übernimmt der Arbeitgeber in Nettolohnabreden für Minijobber ausdrücklich die Zahlung der pauschalen Sozialversicherungsbeiträge, so findet formell zwar keine Verlagerung der Sozialabgaben auf die Beschäftigten statt. Im Ergebnis werden jedoch bei einer Nettoentlohnung die Sozialabgaben herausgerechnet, sodass durch die geringere Entlohnung de facto sehr wohl eine Abwälzung auf die Beschäftigten stattfindet. ${ }^{\boxplus}$ Rechtfertigungsversuche, es handele sich dabei nur um eine formelle Ungleichbehandlung, im Ergebnis sei Gleichbehandlung gegeben („Ergebnisgleichheit“, vgl. Thüsing 2005, S. 124), können nicht überzeugen. Die Bruttovergütung spiegelt den Wert der Arbeitsleistung wider, ${ }^{(1)}$ eine Ungleichbehandlung ist somit gegeben, wenn geringfügig Beschäftigten nicht das gleiche Bruttoentgelt gezahlt wird. Diese Ungleichbehandlung ist nicht zu rechtfertigen, da sie ein unzulässiges Umgehungsgeschäft darstellt. Nettolohnabreden diskriminieren somit Minijobber und sind wegen unzulässiger Umgehung von $\$ 32$ SGB I daher weder als tarifvertragliche noch als individualrechtliche Vereinbarungen zulässig. Aus dem gleichen Grund sind auch Entgeltregelungen nicht zulässig, die sich zwar am Bruttostundenentgelt sozialversicherungspflichtiger Beschäftigter orientieren, aber einen Abzug der höheren Beitragsbelastung der Arbeitgeber vom Lohn der Minijobber vorsehen (LAG Düsseldorf v. 3.2.2011 - 5 SA 1351/10; bejahend Hanau 2006, S. 810).

\subsection{Flexibler Einsatz durch Arbeit auf Abruf}

Vertragsgestaltungen, wonach die Arbeitsleistung entsprechend dem Arbeitsanfall zu erbringen ist, sind gem. $\$ 12$ Abs. $1 \mathrm{~S} .1$ TzBfG rechtlich zulässig, sofern arbeitsvertraglich vereinbart. Der Arbeitgeber kann die Leistung einseitig bestimmen. Damit hat die Beschäftigte auf Abruf zur Verfügung zu stehen, ohne dass die Zeit der Einsatzbereitschaft gesondert vergütet wird (Kiene 2010, S. 23). Bei Minijobbern können sich diese Regelungen jedoch als besonders missbrauchsanfällig erweisen, da aufgrund der kurzen Arbeitszeitdauer durch die Möglichkeit der flexiblen Zuweisung der Arbeit bei Erkrankungen oder Feiertagen die Pflicht zur Entgeltfortzahlung umgangen werden kann. Eine solche Praxis ist wegen $\$ 3$ Abs. 1 EFZG jedoch nicht zulässig. Folglich besteht die Pflicht zur Zahlung des Entgelts, wenn nach der bisherigen Praxis eine Einteilung an dem fraglichen Tag zu erwarten gewesen wäre; abzustellen ist dabei auf die Durchschnittsvergütung der letzten drei Monate. Bei längeren Erkrankungen wäre nach der vereinbarten Wochenarbeitszeit abzurechnen (Däubler 2009, Rn. 1964). \$12 Abs. 1 und 2 TzBfG normieren Mindestbedingungen für die Gestaltung der Abrufarbeit. So verpflichtet $\$ 12$ Abs. 2 TzBfG den Arbeitgeber, dem Arbeitnehmer die Lage der Arbeitszeit und die Dauer des jeweiligen

(7) BAG vom 23.10.1991 (Zeitschrift fürTarifrecht 1992, S. 72), vom 1.11.1995 (Arbeitsrechtliche Praxis BeschFG 1985 § 2 Nr. 45, 46), vom 12.06.1996 (5 AZR 960/94), vom 9.10.1996 (AP BeschFG 1985 § 2 Nr. 50); dieses zieht Thüsing (2005, S. 123) jedoch in Erwägung.

8 DHV - Die Berufsgewerkschaft nennt sich diese Mitgliedsorganisation des Christlichen Gewerkschaftsbundes. Vgl. zu der Unzulässigkeit der genannten Tarifverträge das Gutachten von Nebe/Bernhardt 2011.

( Nachteilig für die Leistungsberechtigten ist nicht nur das Entziehen einer Sozialleistung, sondern auch, wenn ihnen eine über gesetzliche Bestimmungen hinausgehende Pflicht aufgebürdet wird (Nebe/Bernhardt 2011, S. 26; Krause 1999, S. 391).

(10 Sofern auch pauschale Abgaben zur Rentenversicherung abgeführt werden. Für vertiefte Hinweise zu steuerrecht lichen Regelungen bei geringfügiger Beschäftigung siehe Nebe/Bernhardt 2011, S. $11 \mathrm{ff}$.

(1) Zur genauen Berechnung, wie viel der Arbeitgeber dabei spart, siehe Nebe/Bernhardt 2011, S. $19 \mathrm{f}$.

(12) Dieser Grundsatz findet sich in BAG v. 12.06.1996 (5 AZR 960/94, AP Nr. 4 zu § 611 BGB Werksstudent); Landesarbeitsgericht Hamm 18.03.2009 (6 Sa 1214/08, Arbeitsrecht im Betrieb 2009, S. 133). 
Einsatzes mindestens vier Tage im Voraus mitzuteilen (Laux/ Schlachter 2011, \$12, Rn. 69).

$\$ 12$ Abs. 1 S. 2 TzBfG schreibt die Festlegung einer bestimmten Dauer der täglichen und wöchentlichen Arbeitszeit vor, wobei die Vorschrift dispositiv ist. Eine Mindesthöhe ist nicht festgeschrieben; lediglich wenn keine Vereinbarung über die Höhe der wöchentlichen Arbeitszeit getroffen wurde, greift die gesetzliche Fiktion aus $\$ 12$ Abs. 1 S. 3 TzBfG, wonach eine Wochenarbeitszeit von zehn Stunden fingiert wird. Und nur falls auch die tägliche Arbeitszeit nicht festgelegt ist, greift S. 4 ein, wonach der Arbeitgeber die Minijobberin mindestens drei Stunden in Folge in Anspruch nehmen muss. Arbeitsverträge können mithin auch vorsehen, dass geringfügig Beschäftigte dem Arbeitgeber variabel fünf Stunden pro Woche, gleichgültig an welchen Tagen und zu welcher Uhrzeit, zur Verfügung stehen, sodass die Arbeitnehmerin für ein äußerst geringes Entgelt letztlich rund um die Uhr arbeitsbereit sein muss. Das BAG erklärte 2005 gar Arbeitsvertragsklauseln für zulässig, die den Arbeitgeber nicht nur zur laufenden einseitigen Veränderung der Arbeitszeitlage, sondern auch zur Veränderung der Arbeitszeitdauer bis zu $25 \%$ berechtigen sollen - und erweitert damit das arbeitszeitbezogene Direktionsrecht (BAG v. 7.12.2005, Arbeit und Recht 2006, S. 170; Decruppe/Utess 2006, S. 347). Eine unangemessene Benachteiligung der geringfügig Beschäftigten im Sinne der $\$ \$ 307 \mathrm{ff}$. BGB wurde nicht angenommen. Diese Rechtsprechung wurde zum Teil extrem begrüßt, ${ }^{(3)}$ was aber nichts daran ändert, dass sie gegen den Wortlaut von $₫ 12$ Abs. 1 S. 2 TzBfG verstößt, der auf die Vereinbarung einer „bestimmte(n) Dauer“, nicht aber einer Mindestdauer abstellt. Insoweit ist den Stimmen der Literatur zuzustimmen, die aufgrund der Notwendigkeit der Vereinbarung einer „bestimmten Dauer“ der Arbeitszeit keinen Raum für einen durch den Arbeitgeber frei bestimmbaren Teil sehen und eine noch stärkere Verlagerung des Wirtschaftsrisikos auf die Beschäftigten ablehnen (Laux/Schlachter 2011, 12 TzBfG, Rn. 43; kritisch auch Arnold 2007, S. 6).

Umstritten ist ferner, welcher Referenzrahmen für die Erbringung der Arbeitszeit zugrunde zu legen ist. Folgt man dem BAG nicht dahingehend, dass die Festlegung einer Mindestdauer ausreichend ist, so stellt sich die Frage, ob der Bezugsrahmen von einer Woche zwingend ist, oder ob auch auf den monatlichen oder gar Jahresdurchschnitt abgestellt werden kann. Letzteres wird z.T. mit dem Argument bejaht, der Gesetzgeber habe lediglich auf die regelmäßige Dauer der wöchentlichen und täglichen $\mathrm{Ar}$ beitszeit Bezug nehmen wollen, um so die Höhe der Vergütungspflicht des Arbeitgebers festlegen zu können. Dies schließe nicht aus, dass die tatsächliche Arbeitszeit in einem größeren Bezugsrahmen verteilt werde. ${ }^{(6)}$ Argumentiert wird damit, dass bei anderer Auslegung jede Möglichkeit der Flexibilisierung der Arbeitszeit in diesem Anwendungsbereich ausgeschlossen sei (Meinel et al. 2009, $\$ 12 \mathrm{Rn} 23)$.
Dieses Argument greift zu kurz, da der Gesetzgeber mit Schaffung der Norm gerade einen Schutz der Arbeitnehmer vor ausufernder Flexibilisierung beabsichtigte. Dieses zeigt nicht nur die Wortwahl von Satz 2 („die Vereinbarung muss eine bestimmte Dauer der wöchentlichen und täglichen Arbeitszeit festlegen“), sondern auch die Gesamtkonstruktion von $\$ 12$ Abs. 1 TzBfG, wonach eine gesetzliche Fiktion von zehn Stunden pro Woche und mindestens drei zusammenhängenden Stunden greift, wenn die Parteien abweichend von Satz 2 keine vertragliche Absprache getroffen haben. Sowohl Aufbau als auch Wortwahl machten keinen Sinn, wenn der Gesetzgeber lediglich Bezug auf einen Referenzrahmen hätte nehmen wollen. Der Gegenauffassung ist daher zuzustimmen, die auf den mit Einführung des TzBfG geänderten Wortlaut verweist, der in $₫ 12$ Abs. 1 S. 2 und 3 TzBfG, im Unterschied zum Beschäftigungsförderungsgesetz (BeschFG), eindeutig auf die wöchentliche Arbeitszeit abstellt. ${ }^{\text {(1) Ein }}$ längerer Bezugsrahmen würde das einseitige Bestimmungsrecht des Arbeitgebers über die Arbeitszeitlage noch stärker erweitern, was durch die festgelegte Dauer gerade verhindert werden soll.

Monats- oder Jahresarbeitszeitkonten sind dennoch möglich, da tarifliche Regelungen nach $₫ 12$ Abs. 3 TzBfG auch Regelungen zuungunsten der Arbeitnehmer festlegen und folglich einen größeren Bezugsrahmen vorsehen können. Je weiter der Bezugsraum gefasst ist, desto stärker wird allerdings die Dispositionsmöglichkeit der Arbeitnehmerin eingeschränkt. Auch bei einem wöchentlichen Bezugsrahmen ist bereits ein derart flexibler Einsatz geringfügig Beschäftigter möglich, dass die Ausgewogenheit von Leistung und Gegenleistung gefährdet sein kann, stehen den Vorteilen des Arbeitgebers auf Arbeitnehmerseite doch ausschließlich Nachteile gegenüber: Der Arbeitnehmer wird auf Kosten seiner zeitlichen und finanziellen Souveränität mit dem Beschäftigungsrisiko des Arbeitgebers belastet (Kiene 2010, S. 25). Zudem ist es bei einer solchen Vertragskonstruktion unmöglich, einer weiteren Tätigkeit nachzugehen. Abrufarbeit bewirkt somit eine Verfestigung des geringen Arbeitsvolumens und hält die Beschäftigten damit in Armut, sofern im Haushalt kein weiteres Einkommen verfügbar ist.

\footnotetext{
(13 Die positive Kommentierung findet sich etwa bei Preis/ Lindemann 2006; Preis 2011b, Rn. 23; Schmitt-Rolfes 2006; Bauer/Günther 2006 und Hohenstatt/Schramm 2007.

(4) So etwa Meinel et al. 2009, § 12TzBfG, Rn. 22, 27; Laux/ Schlachter 2011, § 12TzBfG, Rn. 53; Preis 2011b, Rn. 22 und Worzalla et al. 2001, § 12TzBfG, Rn. 39.

(5) In diesem Sinne Buschmann et al. 2001, § 12TzBfG, Rn. 66; Däubler 2001, S. 222; Schiek 2002, S. 34; Boewer 2008, TzBfG § 12, Rn. 13; Kittner et al. 2011, § 12TzBfG, Rn. 9, 13, 19; Mühlmann 2006 , S. 359; wohl auch LAG Düsseldorf v. 30.08.2002 (Neue Zeitschrift für Arbeitsrecht-Rechtsprechungsreport 2003, S. 407).
} 


\section{Regulierung durch Betriebs- und Tarifvertragsparteien}

Untersuchungen zeigen, dass geringfügige Beschäftigung seltener in Betrieben zu finden ist, die durch Tarifbindung in regulierte Arbeitsbeziehungen einbezogen sind (Klenner et al. 2009, S. 213). Auch wenn ein Betriebsrat im Betrieb tätig ist, ist die Quote geringfügiger Beschäftigung im Betrieb niedriger. ${ }^{\circledR}$ Betriebs- und Tarifvertragsparteien steht ein vielfältiges Instrumentarium zum Schutz von Minijobbern zur Verfügung.

\subsection{Betriebsverfassungsrechtliche Handlungsmöglichkeiten}

Betriebsverfassungsrechtlich werden geringfügig Beschäftigte wie Vollzeitbeschäftigte behandelt, sie werden daher bei der Ermittlung der Betriebsgröße mitgerechnet ${ }^{\circledR}$ und sind zum Betriebsrat sowohl aktiv als auch passiv wahlberechtigt. Dennoch schöpfen Betriebsräte das ihnen betriebsverfassungsrechtlich zur Verfügung stehende Instrumentarium zum Schutz von Minijobbern oftmals nicht aus. Diese Beschäftigten sind aufgrund des geringen Arbeitsvolumens weniger als die Stammbelegschaft im Betrieb anwesend und nehmen seltener an Betriebsversammlungen teil; auch die Sprechstunden des Betriebsrats suchen sie weniger häufig auf. Daher erreicht der Betriebsrat diese Beschäftigtengruppe nicht automatisch; es bedarf vielmehr gezielter Strategien, die zudem die spezifischen Bedürfnisse berücksichtigen. Eine Untersuchung aus dem sächsischen Einzelhandel deutet an, dass Betriebsräte häufig wenig Unterstützung bei den Vollzeitkräften finden, wenn sie sich für geringfügig Beschäftigte einsetzen. Unter Umständen wird die Personalkostensenkung durch prekär Beschäftigte von der Stammbelegschaft unter dem Gesichtspunkt des zusätzlichen Schutzes für den eigenen Arbeitsplatz gesehen (Benkhoff/Hermet 2008, S. 5, 15) und es gelingt nicht, Spaltungslinien im Betrieb aufzulösen (siehe dazu Hinz in diesem Heft). Der Betriebsrat hat jedoch die Aufgabe, Diskriminierung zu bekämpfen und dabei die Interessen aller Beschäftigten zu vertreten.
Der Betriebsrat hat in vielerlei Hinsicht die Möglichkeit, zugunsten geringfügig Beschäftigter tätig zu werden. So greift das Mitbestimmungsrecht auch hinsichtlich der Arbeitszeit von Teilzeitbeschäftigten. Gemäß $\$ 87$ Abs. 1 Nr. 2 Betriebsverfassungsgesetz (BetrVG) ist der Betriebsrat hinsichtlich Beginn und Ende der täglichen Arbeitszeit und der Verteilung auf die einzelnen Wochentage zu beteiligen, sofern ein kollektiver Tatbestand gegeben ist. Der Betriebsrat ist somit zu beteiligen, wenn Arbeit auf Abruf eingeführt werden soll, kraft Mitbestimmung kann er dieses Arbeitszeitsystem auch ausschließen ${ }^{\circledR}$ - mit dem bekannten Prozedere des Einigungsstellenverfahrens. Auch die Frage einer täglichen Mindestarbeitszeit, einer Höchstzahl von Arbeitstagen pro Woche und des Einsatzrahmens obliegt somit nicht allein der arbeitgeberseitigen Entscheidung oder der vertraglichen Ausgestaltung der Parteien. Lediglich die Mindestdauer der wöchentlichen Arbeitszeit wird allein von den Arbeitsvertragsparteien festgelegt, ${ }^{\circledR}$ auch der jeweilige individuelle Abruf unterliegt nicht der Mitbestimmung. ${ }^{20}$ Der Betriebsrat kann Flexibilität und Verfügbarkeit von Minijobbern durch den Abschluss einer (erzwingbaren) Betriebsvereinbarung begrenzen und Regularien zur Gleichbehandlung (beispielsweise in Bezug auf Weiterbildung) festschreiben, auch der Vorrang bei der Besetzung von Vollzeitarbeitsplätzen kann normiert werden. ? $^{2}$

Gemäß $\$ 99$ BetrVG ist der Betriebsrat zudem bei personellen Einzelmaßnahmen (auch) in Bezug auf geringfügig Beschäftigte zu beteiligen. In Unternehmen mit mehr als 20 wahlberechtigten Arbeitnehmern muss er gemäß Absatz 1 vor jeder Einstellung, Ein- und Umgruppierung sowie Versetzung unterrichtet werden und kann die Zustimmung zur geplanten Maßnahme verweigern, sofern eines der Katalogmerkmale des Absatz 2 erfüllt ist. Nummer 1 bietet ein Zustimmungsverweigerungsrecht, wenn die geplante Maßnahme gegen ein Gesetz, eine Verordnung, Unfallverhütungsvorschrift oder gegen Bestimmungen aus Tarifvertrag oder Betriebsvereinbarung verstößt. Infrage kommt daher bei der Einstellung geringfügig Beschäftigter ${ }^{2}$ ein Verstoß gegen die Regelungen zur Abrufarbeit aus $\$ 12$ Abs. 1, 2 TzBfG. Das BAG unterscheidet jedoch zwischen der Maßnahme als solcher und separat zu betrachtenden Ver-
(16) Wobei dies auch abhängig von Branche und Betriebsgröße ist vgl. Pfeifer 2007, S. 75; Klenner et al. 2009, S. 216, 224

(1) Die Betriebsgröße ist gemäß $§ 1$ Betriebsverfassungsgesetz (BetrVG) für die Errichtung eines Betriebsrats und für die Zahl seiner Mitglieder nach § 9 BetrVG maßgeblich.

(1) BAG v. 13.10.1987, v. 28.09.1988, v. 16.07.1991 (AP Nr. 24, 29, 44 zu § 87 BetrVG 1972 Arbeitszeit); Däubler et al. 2010, § 87 BetrVG, Rn. 85; Fitting et al. 2010, § 87 Rn. 124; Buschmann et al. 2001, § 12TzBfG, Rn. 85.

(1) Anderer Ansicht sind Däubler et al. (2010, § 87 BetrVG Rn. 85), die auch hier ein zwingendes Mitbestimmungsrecht als gegeben ansehen.
20 So urteilte das BAG am 28.9.1988 (Neue Zeitschrift für Arbeitsrecht 1989, S. 185) und am 13.10.1987, NZA 1988, S. 254; siehe auch Kittner et al. 2011, § 12TzBfG, Rn. 33; Preis 2011b, Rn. 36; Buschmann et al. 2001, § 12 TzBfG, Rn. 85 sowie Däubler 2006, Rn. 1665.

(2) Auswahlrichtlinien, die Minijobbern Vorrang bei der Besetzung von Vollzeitstellen gewähren, unterliegen gem. $\S 95$ BetrVG der Mitbestimmung des Betriebsrats.

22 Gleiches gilt bei einer Versetzung, d.h. wenn ein vormals versicherungspflichtiges Arbeitsverhältnis in geringfügige Beschäftigung umgewandelt wird und sich dadurch die Art derTätigkeit ändert. 
tragsbestandteilen. Die Zustimmung soll der Betriebsrat nur verweigern können, wenn die vorgesehene Beschäftigung selbst gegen eine Rechtsvorschrift verstößt, ${ }^{3}$ nicht aber, wenn Festlegungen des Arbeitsvertrags gegen rechtliche Bestimmungen verstoßen. Wurde allerdings bei $\mathrm{Ab}$ rufarbeit (entgegen $\$ 12$ Abs. 2 TzBfG) vereinbart, dass keine Ankündigungsfrist einzuhalten ist, der Arbeitnehmer also auch am Folgetag eingesetzt werden kann, so ist darin die Absicht des Arbeitgebers zu erkennen, ihn in einer vom Gesetzgeber missbilligten Art und Weise einzusetzen, sodass die Zustimmungsverweigerung greift (Däubler et al. 2010, $\$ 99$, Rn. 173, 175). Gleiches gilt auch, wenn der Arbeitgeber den Minijobber entgegen zeitlichen Begrenzungen, die in einer Betriebsvereinbarung oder tarifvertraglich vereinbart wurden, einsetzen will oder wenn gegen das Diskriminierungsverbot aus $₫ 4$ Abs. 1 TzBfG verstoßen würde. $\$ 99$ BetrVG weist dem Betriebsrat zudem die Kontrolle darüber zu, ob der Arbeitnehmer nach dem einschlägigen Tarifvertrag korrekt eingruppiert wurde. Insbesondere an diesem Punkt besteht großer Handlungsbedarf, da Berichte aus der Praxis zeigen, dass geringfügig Beschäftigte häufig außerhalb des tarifvertraglichen Lohngefüges oder unterhalb der einschlägigen Tarifgruppe entlohnt werden (Voss-Dahm 2009).

Der Betriebsrat kann ferner die Zustimmung nach $\$ 99$ Abs. 2 Nr. 3 verweigern, wenn die begründete Besorgnis besteht, dass durch die geplante Maßnahme im Betrieb beschäftigte Arbeitnehmer Nachteile erleiden, z.B. wenn sich die Arbeit der Stammbelegschaft infolge veränderter Arbeitsaufteilung verdichten oder anders erschweren würde. Verweigert der Betriebsrat die Zustimmung zur geplanten Maßnahme, muss der Arbeitgeber diese vom Arbeitsgericht ersetzen lassen ( $\$ 99$ Abs. 4 BetrVG).

Dem Betriebsrat ist es zudem möglich, geringfügig Beschäftigte umfassend über ihre Rechte aufzuklären und auf die rentenrechtliche Aufstockungsmöglichkeit hinzuweisen. Er hat zudem die Möglichkeit, auf der Grundlage von Informationen nach $₫ 92$ BetrVG Personalprozesse in Gang zu setzen, ${ }^{3}$ um diskriminierende Praktiken gegenüber geringfügig Beschäftigten zu ermitteln; Betriebsanalysen können erstellt werden. Systematische Personalstatistiken, Bedarfserhebungen und Monitoring-Instrumente fehlen bislang weitgehend (Maschke/Wiechmann 2010, S. 487, 491), bieten aber einen guten Ausgangspunkt für gezieltes Handeln zugunsten prekär Beschäftigter.

\subsection{Handlungsmöglichkeiten der Tarifvertragsparteien}

Regelungen zur Dauer und Lage der Arbeitszeit gehören zu den Kernbereichen der nach Art. 9 Abs. 3 Grundgesetz (GG) durch die Tarifvertragsparteien regelbaren Arbeitsund Wirtschaftsbedingungen. Die Tarifvertragsparteien haben somit die Möglichkeit, die Flexibilisierung geringfügiger Beschäftigung zu begrenzen, indem Regeln für ihren zeitlichen Einsatz in Tarifverträge aufgenommen werden.
Bei der Regelung von Abrufarbeit können die Tarifvertragsparteien mitbestimmungspflichtige Angelegenheiten selbst regeln, da $₫ 87$ Abs. 1 S. 1 einen Vorrang tariflicher Regelungen normiert.

Da die gesetzlichen Regelungen zur Arbeit auf Abruf tarifdispositiv ausgestaltet sind ( $\$ 12 \mathrm{Abs} .3 \mathrm{TzBfG}$ ), kann von $\$ 12$ Abs. 1 und 2 TzBfG durch Tarifvertrag auch zuungunsten der Arbeitnehmer abgewichen werden. ${ }^{3}$ Tarifverträge dürfen allerdings nicht gegen $₫ 4$ TzBfG verstoßen, da $\$ 22$ TzBfG das Diskriminierungsverbot ausdrücklich nicht zur Disposition der Tarifvertragsparteien stellt. ${ }^{\text {(2) }}$ Minijobber können somit nicht von tarifvertraglichen Leistungen ausgeschlossen werden, auf die alle anderen Beschäftigten einen Anspruch haben. ${ }^{27}$

$\$ 12$ Abs. 3 S. 2 TzBfG ermöglicht es, dass nicht tarifgebundene Arbeitgeber über individualvertragliche Bezugnahmeklauseln die tariflichen Regelungen anwenden, sofern sie sich im Geltungsbereich eines (zulässigen) Tarifvertrags befinden.

\subsubsection{Regelungen zum Schutz der Beschäftigten}

Insbesondere angesichts der zum Teil ausufernden Flexibilität zulasten der Beschäftigten bei Arbeit auf Abruf ist es sinnvoll, dass die Tarifvertragsparteien Regelungen über die wöchentliche und tägliche Arbeitszeit der auf Abruf Beschäftigten treffen. Möglich wäre beispielsweise das Festlegen einer täglichen Mindest- oder Höchsteinsatzzeit. Anregung dafür bieten die Regelungen aus $\$ 12$ Abs. 1 und 2 TzBfG, die Tarifvertragsparteien können aber auch darüber hinausgehen, indem sie beispielsweise eine Mindesteinsatzzeit von vier Stunden festlegen. Auch die Festlegung einer durchschnittlichen wöchentlichen Arbeitszeit in Kombination mit einem Arbeitszeitkonto kann durch Tarifvertrag getroffen werden.

23. So die Urteile des BAG vom 16.7.1985, AP Nr. 21 zu § 99 BetrVG 1972 (Anmerkung Kraft) und vom 18.10.1988, Entscheidungssammlung zum Arbeitsrecht § 99 BetrVG 1972 Nr. 69.

24 Diese müssen sich gerade auch auf gleichstellungsspezifische Aspekte stützen (§ 92 Abs. 3 BetrVG).

(25) Abweichungen von gesetzlichen Normen zugunsten der Arbeitnehmer sind nach dem Günstigkeitsprinzip ohnehin immer möglich.

20 So urteilte das BAG am 28.7.1992 (3 AZR 173/92) zu § 2 Abs. 1 BeschFG: Zusatzversorgung Teilzeitbeschäftigter.

(27) Da die ungleiche Behandlung nur an der niedrigeren Stundenzahl ansetzen könnte, ist eine sachliche Rechtfertigung nicht möglich.

23 § 12 Abs. 3TzBfG ermöglicht den Tarifvertragsparteien auch ein Abweichen zuungunsten der Arbeitnehmer. 
Ebenso steht es den Tarifvertragsparteien offen, zu vereinbaren, dass Abrufarbeit oder geringfügige Beschäftigung insgesamt nicht zum Einsatz kommt. Allerdings besteht in diesem Fall die Gefahr, dass das Problem nur verlagert wird, indem zwar die tarifgebundenen Unternehmen selbst keine geringfügig Beschäftigten einsetzen, der Einsatz von Minijobbern im Unternehmen aber über (nicht tarifgebundene) Personaldienstleister erfolgt.

Entgelt und andere tarifvertragliche Leistungen stehen Minijobbern aufgrund des Diskriminierungsverbots ohnehin anteilig ihrer Stundenzahl zu. Die Tarifvertragsparteien können dieses jedoch zur Klarstellung im Tarifvertrag aufführen und zudem Regelungen wie die Zahlung von Überstundenzuschlägen $a b$ der ersten Stunde für geringfügig Beschäftigte vorsehen.

\subsubsection{Regelungen zuungunsten der Arbeitnehmer}

$\$ 12$ Abs. 3 TzBfG stellt das Abweichen vom gesetzlichen Modell zuungunsten der Arbeitnehmer unter die Voraussetzung, dass der Tarifvertrag überhaupt Regelungen über die tägliche und wöchentliche Arbeitszeit und die Vorankündigungsfrist vorsieht. Es ist daher nicht zulässig, dass die Tarifvertragsparteien die Dauer der Arbeitszeit offen lassen und dem Arbeitgeber ein einseitiges Bestimmungsrecht gewähren. An einer „Regelung“ der wöchentlichen Arbeitszeit fehlt es auch, wenn eine Bandbreite von 0 - 40 Std./Woche vereinbart wird (Laux/Schlachter 2011, 12 TzBfG, Rn. 81, 85). Der Tarifvertrag kann zudem nur Entscheidungen über die Ausgestaltung der Abrufarbeit für solche Arbeitsverhältnisse treffen, in denen sich die Vertragsparteien auf Abrufarbeit geeinigt haben. Wurden arbeitsvertraglich jedoch feste Arbeitszeiten und Arbeitsdauer vereinbart, dann tritt eine tarifvertragliche Regelung, die es dem Arbeitgeber gestattet, den vertraglich festgeschriebenen Stundenumfang zu unterschreiten oder die Arbeitszeit entsprechend dem Arbeitsanfall flexibel zu verteilen, unter dem Gesichtspunkt der Günstigkeit zurück (Kiene 2010, S. 182, 184). Die Tarifvertragsparteien können zudem die in $₫ 12$ Abs. 2 TzBfG festgelegte Mindestankündigungsfrist unterschreiten, wobei die festgelegte Frist dem Arbeitnehmer zumutbar sein muss und die Grenzen zur (vergütungspflichtigen) Rufbereitschaft nicht völlig unterlaufen werden dürfen (Laux/Schlachter 2011, \$12 TzBfG, Rn. 88). Tarifverträge allerdings, die erkennbar nicht dem Interesse der Beschäftigten entsprechen, weil sie von tarifdispositivem Gesetzesrecht nach unten abweichen, ohne entsprechende kompensatorische Leistungen vorzusehen, führen die eigentliche Funktion der Tarifautonomie ad absurdum, da sie diese zum Absenken von Standards missbrauchen. Ihre eigentliche Aufgabe, die Interessen ihre Mitglieder $\mathrm{zu}$ vertreten, nehmen solche Scheintarifverträge nicht wahr (Zimmer 2011, S. 25, 30 f.; Schlachter/Klauk 2010, S.354 ff.).

\section{Fazit}

Geringfügige Beschäftigung ist aufgrund der sozialrechtlichen Sonderstellung strukturell besonders missbrauchsanfällig und bietet dem Arbeitgeber Anreize, Vollzeitarbeitsverhältnisse in mehrere Minijobs aufzuspalten. Die Beschäftigten hingegen wünschen sich oftmals ein höheres Arbeitsvolumen. Auch aus diesem Grund sollte diese Beschäftigungsform abgeschafft werden und „normaler“ Teilzeitarbeit Platz machen. Der 68. Deutsche Juristentag nahm den Vorschlag des Gutachters Waltermann (2010) an, die sozialrechtliche Privilegierung geringfügiger Beschäftigung sowie die sogenannte „Gleitzone“ ( $\$ 20$ Abs. 2 SGB IV) abzuschaffen. Frauenverbände erheben diese Forderung bereits seit Langem, zementiert doch die Kombination von geringfügiger Beschäftigung und Ehegattensplitting eine traditionelle Rollenverteilung in der Ehe, bei der Frauen die Verliererinnen sind. ${ }^{2}$

Notwendig wäre, dass der Gesetzgeber tätig wird. Geschieht dies vorerst nicht, bleiben nur die Betriebs- und Tarifvertragsparteien als Akteure, um den Status geringfügig Beschäftigter zu verbessern, unfreiwillige Flexibilität zu begrenzen und Diskriminierung zu verhindern.

29 Auch der erste Gleichstellungsbericht der Bundesregierung spricht sich entschieden für die Abschaffung dieser Beschäftigungsform aus (BT-Drs. 17/6240, S. 155).

\section{LITERATUR}

Arnold, M. (2007): Änderungsvorbehalte zur Arbeitszeitdauer, in: Rieble, V. (Hrsg.): Festschrift für Manfred Löwisch: zum 70. Geburtstag, München, S. 1-15

Bundesagentur für Arbeit (BA) (2010): Erwerbstätige Arbeitslosengeld-II-Bezieher, Beschäftigungstabellen, http://statistik. arbeitsagentur.de

Bundesagentur für Arbeit (BA) (2011): Arbeitsmarkt in Zahlen. Beschäftigungsstatistik, Nürnberg, http://statistik.arbeitsagentur.de

Bauer, J.-H./Günther, J. (2006): Heute lang, morgen kurz Arbeitszeit nach Maß!, in: Der Betrieb 59 (17), S. 950-952 Benkhoff, B./Hermet, V. (2008): Zur Verbreitung und Ausgestaltung geringfügiger Beschäftigung im Einzelhandel. Eine explorative Studie aus der Perspektive von Management und Beschäftigten, in: Industrielle Beziehungen 15 (1), S. 5-31

Boecken, W./Joussen, J. (2010): Teilzeit- und Befristungsgesetz. Handkommentar, Baden-Baden

Boewer, D. (2008): Teilzeit- und Befristungsgesetz. Kommentar für die Praxis, München

Brehmer, W./Seifert, H. (2008): Sind atypische Beschäftigungsverhältnisse prekär?, in: Zeitschrift für Arbeitsmarktforschung 41 (4), S. 501-531

Buschmann, R./Dieball, H./Stevens-Bartol, E. (2001): Das Recht derTeilzeitarbeit. Kommentar für die Praxis, Frankfurt a. M 
Däubler, W. (2001): Das neue Teilzeit- und Befristungsgesetz, in: Zeitschrift für Wirtschaftsrecht 22 (6), S. 217-225

Däubler, W. (2006): Das Arbeitsrecht 1 - Die gemeinsame Wahrung von Interessen im Betrieb. Leitfaden für Arbeitnehmer, Reinbek bei Hamburg Däubler, W. (2009): Das Arbeitsrecht 2 - Das Arbeitsverhältnis: Rechte und Pflichten, Kündigungsschutz, Reinbek bei Hamburg

Däubler, W./Kittner, M./Klebe, T./Wedde, P. (2010): Betriebsverfassungsgesetz. Kommentar für die Praxis mit Wahlordnung und EBR-Gesetz, Frankfurt a. M.

Decruppe, H./Utess, M. (2006): Arbeitszeitpolitik durch die Judikatur? - Eine kritische Betrachtung der Entscheidung des BAG v. 7.12.2005, in: Arbeit und Recht 54 (10), S. 347-351

\section{Deutsche Rentenversicherung Knappschaft Bahn-See/Minijobzentrale} (2011): Aktuelle Entwicklungen im Bereich der geringfügigen Beschäftigung, Essen, http://www.minijob-zentrale.de/DE/Service/DownloadCenter (letzter Abruf 01.09.2011)

Deutscher Frauenrat (2010): Minijobs - Wege in die Armut: Kann sich unsere Gesellschaft Minijobs leisten?, Positionspapier zur Mitgliederversammlung, Berlin

Feldhoff, K. (2009): Frauenarbeit im Einzelhandel: Geringfügig beschäftigt gering entlohnt - gering geschätzt?! Anmerkungen zum Urteil des LAG Hamm vom 18.03.2009 - 6 SA 282/08, in: Streit - Feministische Rechtszeitschrift 26 (3), S. 99-107

Fitting, K./Engels, G./Schmidt, I./Trebinger, Y./Linsenmaier, W. (2010): Betriebsverfassungsgesetz - BetrVG, München

Hanau, P. (2005): Gleichbehandlung geringfügig Beschäftigter beim Entgelt, in: Der Betrieb 58 (17), S. 946-948

Hanau, P. (2006): Das Rätsel Minijob, in: Neue Zeitschrift für Arbeitsrecht 23 (15), S. 809-811

Hohenstatt, K. S./Schramm, N. (2007): Neue Gestaltungsmöglichkeiten zur Flexibilisierung der Arbeitszeit, in: Neue Zeitschrift für Arbeitsrecht 24 (5), S. $238-242$

Hromadka, W. (2001): Befristete und bedingte Arbeitsverhältnisse neu geregelt, in: Betriebs-Berater 56 (12), S. 674-677

Kalina, T./Weinkopf, C. (2008): Weitere Zunahme von Niedriglohnbeschäftigung, IAQ-Report (1), Duisburg

Kiene, W. (2010): Arbeit auf Abruf i. S. d. § 12 Absatz 1 Satz 1 TzBfG, BadenBaden

Kittner, M./Däubler, W./Zwanziger, B. (2011): KSchR - Kündigungsschutzrecht, Frankfurt a. M.

Klenner, C./Kohaut, S./Höyng, S. (2009): Vollzeit, Teilzeit, Minijobs, in: Projektgruppe GiB (Hrsg.): Geschlechterungleichheiten im Betrieb. Arbeit, Entlohnung und Gleichstellung in der Privatwirtschaft, Berlin, S. 191-270

Kliemt, M. (2001): Der neue Teilzeitanspruch, in: Neue Zeitschrift für Arbeitsrecht 18 (2), S. 63-71

Krause, R. (1999): Abwälzung des Pauschalbeitrags zur Sozialversicherung auf geringfügig Beschäftigte?, in: Arbeit und Recht 47 (10), S. 390-394

Laux, H./Schlachter, M. (2011): Teilzeit- und Befristungsgesetz. Kommentar, München

Maschke, M./Wiechmann, E. (2010): Instrumente und Akteure betrieblicher Gleichstellungspolitik, in: Projektgruppe GiB (Hrsg.): Geschlechterungleichheiten im Betrieb. Arbeit, Entlohnung und Gleichstellung in der Privatwirtschaft, Berlin, S. 485-550

Meinel, G./Heyn, J./Herms, S. (2009): Kommentar zum Teilzeit- und Befristungsgesetz, München

Mühlmann, M. (2006): Flexible Arbeitsvertragsgestaltung - Die Arbeit auf Abruf, in: Recht der Arbeit 59 (6), S. 356-361

Müller-Glöge, R./Preis, U./Schmidt, I. (Hrsg.) (2011): Erfurter Kommentar zum Arbeitsrecht, München

Nebe, K./Bernhardt, U. (2011): Zulässigkeit von tarifvertraglichen Nettolohnvereinbarungen allein für sogenannte "geringfügig entlohnte Beschäftigte", die sich am Nettolohn vergleichbarer nicht geringfügig Beschäftigter orientieren, unveröffentlichtes Gutachten
Nielebock, H. (2001): Neue gesetzliche Regelungen zur Befristung, in: Arbeitsrecht im Betrieb 22 (2), S. 74-78

Pfeifer, C. (2007): Eine theoretische und empirische Analyse der betrieblichen Determinanten von Teilzeitarbeit, Mini- und Midijobs, in: Zeitschrift für Arbeitsmarktforschung 40 (1), S. 65-76

Preis, U. (2011a): § 4TzBfG, in: Müller-Glöge, R./Preis, U./Schmidt, I. (Hrsg.): Erfurter Kommentar zum Arbeitsrecht, München

Preis, U. (2011b): § 12TzBfG, in: Müller-Glöge, R./Preis, U./Schmidt, I. (Hrsg.): Erfurter Kommentar zum Arbeitsrecht, München

Preis, U./Lindemann, V. (2006): Änderungsvorbehalte - Das BAG durchschlägt den gordischen Knoten, in: Neue Zeitschrift für Arbeitsrecht 23 (12), S. $632-638$

Richardi, R./Wlotzke, O./Wißmann, H./Oetker, H. (Hrsg.) (2009): Münchener Handbuch zum Arbeitsrecht, München

Rolfs, C. (2001), Das neue Recht der Teilzeitarbeit, in: Recht der Arbeit 54 (3), S. $129-143$.

Sachverständigenkommission zur Erstellung des Ersten Gleichstellungsberichtes der Bundesregierung (2011): Neue Wege - Gleiche Chancen. Gleichstellung von Frauen und Männern im Lebensverlauf. Gutachten der Sachverständigenkommission an das Bundesministerium für Familie, Senioren, Frauen und Jugend für den Ersten Gleichstellungsbericht der Bundesregierung, Essen/München

Schiek, D. (2002): Das Teilzeit- und Befristungsgesetz. Neue Paradigmen der Regulierung sogenannter atypischer Beschäftigung, in: Kritische Justiz 35 (1), S. $18-44$

Schmitt-Rolfes, G. (2006): Bedarfsarbeit wieder erlaubt, in: Arbeit und Arbeitsrecht 61 (5), S. 319-324

Statistisches Bundesamt (2009): Niedrigeinkommen und Erwerbstätigkeit, Begleitmaterialien zum Pressegespräch am 19.08. in Frankfurt a. M.,

http://www.destatis.de (Letzter Abruf 01.09.2009)

Thüsing, G. (2005): Ungleichbehandlung geringfügig Beschäftigter in Tarifverträgen, in: Zeitschrift fürTarifrecht 19 (3), S. 118-129

Voss-Dahm, D. (2009): Über die Stabilität sozialer Ungleichheit im Betrieb: Verkaufsarbeit im Einzelhandel, Berlin

Waltermann, R. (2010): Abschied vom Normalarbeitsverhältnis? Welche arbeits- und sozialrechtlichen Regelungen empfehlen sich im Hinblick auf die Zunahme neuer Beschäftigungsformen und die wachsende Diskontinuität von Erwerbsbiographien?, Gutachten für den 68. Deutschen Juristentag, Bonn Wanger, S. (2011): Viele Frauen würden gerne länger arbeiten, IAB-Kurzbericht (9), Nürnberg

Wolf, E. (2002): Lower Wage Rates for Fewer Hours? A Simultaneous WageHours Model for Germany, in: Labour Economics 9 (5), S. 643-663 Worzalla, M./Mailänder, U./Worch, A./Heise, D./Will, P. (2001): Teilzeitarbeit und befristete Arbeitsverträge, München

Zimmer, R. (2010): Geringfügige Beschäftigung von Frauen - ein prekärer Zustand, in: Hohmann-Dennhardt, C./Körner, M./Zimmer, R. (Hrsg.): Geschlechtergerechtigkeit. Festschrift für Heide Pfarr, Baden-Baden, S. 296-310 Zimmer, R. (2011): Eingriff in Gewerkschaftsrechte durch Aufbau vom Arbeitgeber abhängiger Scheingewerkschaften, in: Zimmer, R. (Hrsg.): Rechtsprobleme der tariflichen Unterbietungskonkurrenz, Düsseldorf, S. 25-43

\section{AUTORIN}

REINGARD ZIMMER, Dr., ist Vertretungsprofessorin für Arbeitsrecht an der Universität Hamburg. Arbeitsschwerpunkte: Prekäre Beschäftigungsformen, Tarif- und Arbeitskampfrecht, soziale Mindeststandards, europäisches und internationales kollektives Arbeitsrecht.

reingard.zimmer@wiso.uni-hamburg.de 